\section{REVIEW ARTICLE}

\author{
L.C. Hygino da Cruz Jr \\ I. Rodriguez \\ R.C. Domingues \\ E.L. Gasparetto \\ A.G. Sorensen
}

\title{
Pseudoprogression and Pseudoresponse: Imaging Challenges in the Assessment of Posttreatment Glioma
}

\begin{abstract}
SUMmARY: The current standard of care for newly diagnosed cases of high-grade glioma is surgical resection followed by RT with concurrent chemotherapy. The most widely used criteria for assessing treatment response are based on a 2D measurement of the enhancing area on MR imaging known as the Macdonald Criteria. Recently, nontumoral increases (pseudoprogression) and decreases (pseudoresponse) in enhancement have been found, and these can confuse outcome evaluation. Here we review pseudoprogression and pseudoresponse and describe how better understanding of these phenomena can aid interpretation.
\end{abstract}

G $\mathrm{BM}$ is the most common primary malignant type of brain neoplasm in adults and is associated with a dismal prognosis. The current standard of care is surgical resection followed by RT and concomitant and adjuvant TMZ chemotherapy. This is a relatively recent standard, with pivotal data published in 2005, and it represents a milestone, because this approach has been shown to prolong the overall survival of these patients. ${ }^{1}$ With the standardization of treatment around surgery/RT/TMZ, certain patterns are beginning to emerge that were not previously noticed. In addition, in May 2009, the US Food and Drug Administration approved bevacizumab for recurrent glioblastoma. This anti-VEGF agent also can have a marked pattern of change on MR imaging. In addition to impacting individual patient care, these changes have also had an impact on clinical trials of new therapies.

\section{Macdonald Criteria}

The Macdonald Criteria ${ }^{2}$ are currently the most widely used guideline for assessing response to therapy in patients with highgrade gliomas. These are based on 2D tumor measurements made in MR imaging scans, in conjunction with clinical assessment and corticosteroid dose. According to the Macdonald Cri-

From the Department of Radiology (L.C.H.C.), Clinics IRM, Clinica de Diagnóstico por Imagen and Multi-Imagem, Federal University of Rio de Janeiro, Rio de Janeiro, Brazil; Imagen Médica Avanzada (I.R.), Madrid, Spain; Clinics Clinica de Diagnóstico por Imagen and Multi-Imagem (R.C.D.), Rio de Janeiro, Brazil; Department of Radiology (E.L.G.), Clinics Clinica de Diagnóstico por Imagen and Multi-Imagem, Federal University of Rio de Janeiro, Rio de Janeiro, Brazil; and Department of Radiology, (A.G.S.), Athinoula A. Martinos Center for Biomedical Imaging, Massachusetts General Hospital and Harvard Medical School, Charlestown, Massachusetts.

Please address correspondence to L. Celso Hygino da Cruz Jr, MD, Rua Almirante Saddock de Sá 266, Ipanema, Rio de Janeiro, RJ, CEP:22411-040, Brazil; e-mail: celsohygino@ hotmail.com

Indicates open access to non-subscribers at www.ajnr.org

http://dx.doi.org/10.3174/ajnr.A2397 teria, tumor progression is considered to have occurred when an increase of $>25 \%$ in the size of the contrast-enhancing lesion is observed. There are important limitations to these criteria, which only address the contrast-enhancing component of the tumor, and various updated guidelines for RANO have been published. ${ }^{3,4}$ As radiologists learn early in their training, contrast enhancement in posttreatment brain tumors is nonspecific and may not always be considered a true surrogate of tumor response.

The limitations of the Macdonald Criteria have become acutely apparent with the use of novel treatments. Both pseudoprogression, an increase in the nontumoral enhancing area, and pseudoresponse, a decrease in the enhancing area, show that enhancement by itself is not a measure of tumor activity but rather reflects a disturbed BBB. ${ }^{5}$ Most new RANO criteria, therefore, suggest that the nonenhancing component of the tumor also be taken into account when making assessments about progression or response.

\section{Patterns of Early Enhancement in Posttreatment MR Imaging Follow-Up}

Changes in contrast enhancement detected by MR imaging just after or during treatment can mimic early tumor progression. Increased enhancement can be induced by a variety of nontumoral processes, such as treatment-related inflammation, postsurgical changes, ischemia, subacute radiation effects, and radiation necrosis. ${ }^{6,7}$ Microischemic lesions immediately after surgery and RT can cause BBB disruption. ${ }^{8}$ Therefore, contrast-enhanced MR imaging should be used within the first 48 hours after surgery to identify residual tumor in the postsurgical bed, and no later than 72 hours after surgery to avoid confusion with nontumoral inflammation. Regions with restricted diffusion on an immediately postoperative MR imaging will commonly enhance subacutely for up to 2-3 months after surgery; these are typically thought of as postoperative ischemic changes. In short, residual tumor or 


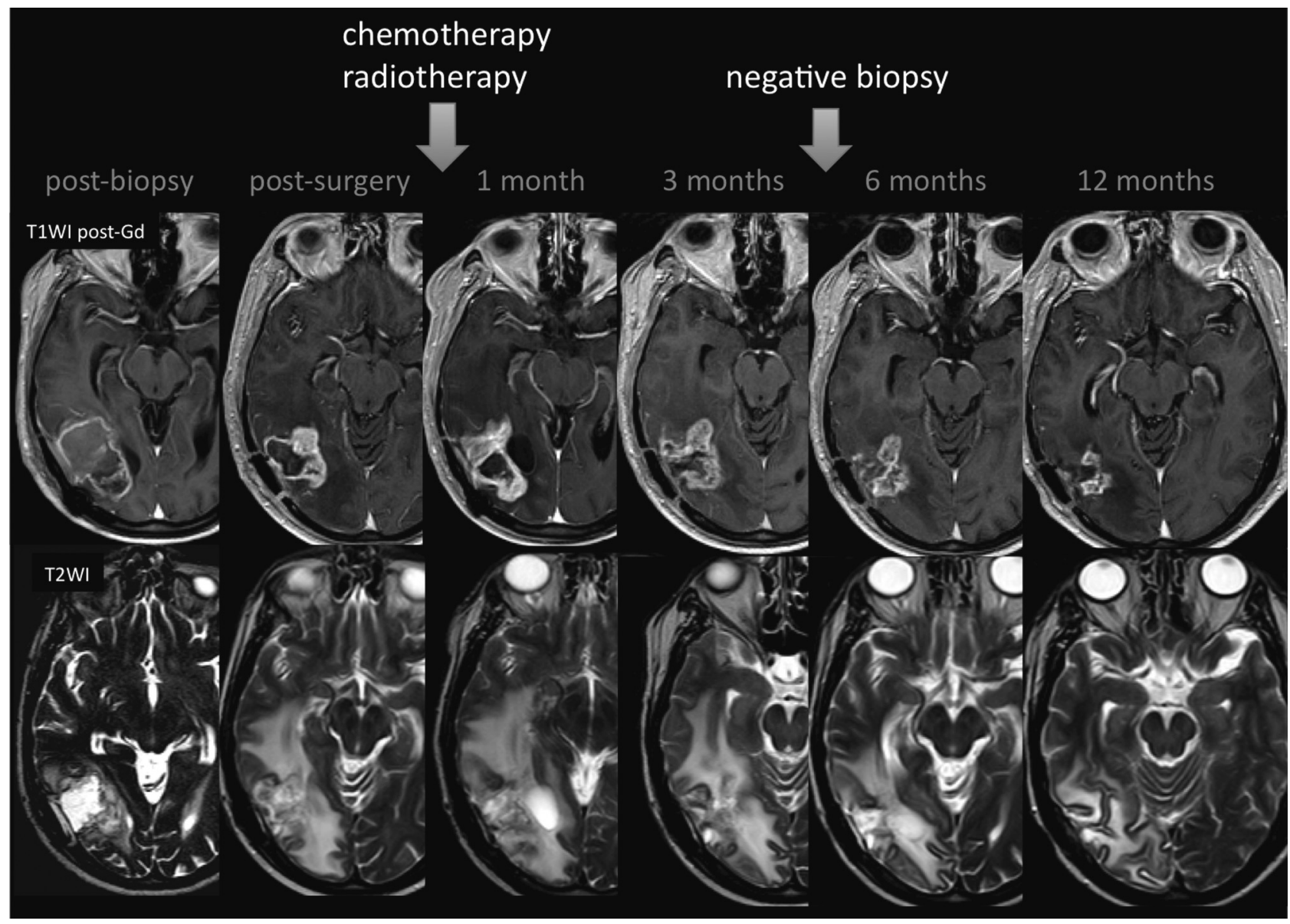

Fig 1. Pseudoprogression. A 59-year-old man with GBM. An MR image obtained 1 month after RT-TMZ demonstrates an expansion of the right temporal lesion. Reductions in both the enhancing portion and the surrounding abnormal hyperintense area in the T2-weighted imaging were seen in the follow-up MR imaging examinations

ischemia sequelae can result in abnormal enhancement on MR imaging follow-up within weeks or months after surgery. ${ }^{7}$

\section{Radiation Injury}

Three different types of effects of brain RT can be identified on the basis of the time of occurrence and clinical presentation: acute (during radiation), subacute or early-delayed (up to 12 weeks after radiation ends), and late (months to years postradiation). Both the acute and subacute types of radiation-induced injury are thought to be caused by vasodilation, disruption of the BBB, and edema. Although diffuse brain swelling has been described, the MR imaging data in the early period after RT usually appear relatively unchanged. ${ }^{9}$ When the subacute type of injury is visible, MR imaging findings can vary from edema to new lesions or increased size of contrast-enhancing lesions within the immediate vicinity of the irradiated tumor volume. ${ }^{6}$ Late delayed reactions, occurring from within a few months to many years, may be due to necrosis triggered by ischemia secondary to blood vessel damage and may depend on increased capillary permeability, leading to fluid transudation and consequent brain edema. More complete study of the time course of these changes and their appearance is urgently needed.

\section{Pseudoprogression}

Shortly after completion of RT, patients with high-grade brain tumors can present with an increase in contrast-enhancing lesion size, followed by subsequent improvement or stabilization without any further treatment (Fig 1). ${ }^{8,9}$ This occurrence, which mimics tumor progression, is termed "pseudoprogression." Pseudoprogression is a subacute treatment-related reaction with or without clinical deterioration. However, in most patients, the increase in radiologic abnormalities is clinically asymptomatic. ${ }^{10}$ Pseudoprogression is most likely induced by a pronounced local tissue reaction with an inflammatory component, edema, and abnormal vessel permeability causing new or increased contrast enhancement on MR imaging examinations. Most important, some studies have found an association between the incidence of pseudoprogression and increased survival; perhaps pseudoprogression represents an active "inflammatory" response against the tumor. ${ }^{9}$

Currently, the only method of distinguishing pseudoprogression and early progression of disease is to perform follow-up examinations of the patients because conventional MR imaging is unable to differentiate the 2 and alternative techniques have not yet been validated in prospective trials. ${ }^{9,11}$ Analysis with follow-up conventional MR imaging examinations allows such a distinction because the changes related to pseudoprogression decrease in size. The development and validation of new methods to evaluate true brain tumor response—as opposed to inflammatory change—would be very helpful in the assessment of treatment efficacy. 


\begin{tabular}{|c|c|c|c|c|c|c|}
\hline \multicolumn{7}{|c|}{ Incidence of pseudoprogression } \\
\hline Publication & $\begin{array}{l}\text { No. of } \\
\text { Patients }\end{array}$ & Response Criteria $^{a}$ & $\begin{array}{c}\text { Criteria } \\
\text { for Early } \\
\text { Progression }^{\mathrm{b}}\end{array}$ & $\begin{array}{l}\text { No. of Early } \\
\text { Progression }\end{array}$ & $\begin{array}{c}\text { Pseudo-Progression } \\
\text { (\% of Early } \\
\text { Progression) }\end{array}$ & $\begin{array}{l}\text { Pseudo-Progression } \\
\text { (\% of Patients) }\end{array}$ \\
\hline Brandes et al ${ }^{11}$ & 103 & $\begin{array}{l}\text { Enhancement increase for earlier } \\
\text { progression than Macdonald criteria }\end{array}$ & 4 Weeks & $50 / 103$ & $32 / 50$ & $32 / 103$ \\
\hline J Clin Oncol $2008^{20}$ & & & & $(48.5 \%)$ & $(64 \%)$ & $(31 \%)$ \\
\hline $\begin{array}{l}\text { Taal et al } \\
\text { Cancer } 2008^{10}\end{array}$ & 85 & Macdonald Criteria & 4 Weeks & $\begin{array}{l}36 / 85 \\
(42 \%)\end{array}$ & $\begin{array}{l}15 / 31^{\mathrm{a}} \\
(48 \%)\end{array}$ & $\begin{array}{c}15 / 85 \\
(17.6 \%)\end{array}$ \\
\hline $\begin{array}{l}\text { Clarke et al } \\
\text { (abstract) J Clin Oncol } 2008^{17}\end{array}$ & 80 & Increased contrast enhancement & Not specified & $\begin{array}{l}33 / 80 \\
(41 \%)\end{array}$ & $\begin{array}{l}8 / 25^{a} \\
(32 \%)\end{array}$ & $\begin{array}{l}8 / 80 \\
(10 \%)\end{array}$ \\
\hline $\begin{array}{l}\text { Gerstner et al } \\
J \text { Neurooncol 2009 }\end{array}$ & 45 & Macdonald Criteria & 17-28 Days & $\begin{array}{l}24 / 45 \\
(53 \%)\end{array}$ & $\begin{array}{l}13 / 24 \\
(54 \%)\end{array}$ & $\begin{array}{l}13 / 45 \\
(29 \%)\end{array}$ \\
\hline $\begin{array}{l}\text { Jefferies et al } \\
\text { abstract Clin Oncol } 2007^{19}\end{array}$ & 15 & Not specified & 6 Months & $\begin{array}{l}9 / 15 \\
(60 \%)\end{array}$ & $\begin{array}{c}3 / 9 \\
(33 \%)\end{array}$ & $\begin{array}{l}3 / 15 \\
(20 \%)\end{array}$ \\
\hline $\begin{array}{l}\text { Chaski et al } \\
\text { Surg Neurol 2009'14 }\end{array}$ & 54 & Increased contrast enhancement & 6 Months & $\begin{array}{l}25 / 54 \\
(46 \%)\end{array}$ & $\begin{array}{l}3 / 25 \\
(12 \%)\end{array}$ & $\begin{array}{c}3 / 54 \\
(5.5 \%)\end{array}$ \\
\hline $\begin{array}{l}\text { Sanghera et al } \\
\text { Can J Neurol Sci } 2010^{16}\end{array}$ & 104 & RECIST & 8 Weeks & $\begin{array}{c}27 / 104 \\
(26 \%)\end{array}$ & $\begin{array}{l}7 / 22^{\mathrm{a}} \\
(32 \%)\end{array}$ & $\begin{array}{c}7 / 104 \\
(7 \%)\end{array}$ \\
\hline $\begin{array}{l}\text { Mangla et al } \\
\text { Radiology } 2010^{15}\end{array}$ & 36 & Macdonald Criteria & 4 Weeks & $\begin{array}{l}19 / 36 \\
(53 \%)\end{array}$ & $\begin{array}{l}7 / 19 \\
(37 \%)\end{array}$ & $\begin{array}{l}7 / 36 \\
(20 \%)\end{array}$ \\
\hline
\end{tabular}

a -All were defined in the text.

${ }^{\mathrm{b}}$ Defined as increase of the enhancing portion of the lesion in the follow-up MR exam.

\section{6-Methylguanine DNA MGMT Promoter}

The methylation status of the MGMT promoter has been shown to be a potent prognostic factor in patients with GBM; cells that are deficient in MGMT have shown an increased sensitivity to TMZ. Patients with low MGMT expression (due to methylation of the promoter) benefit more from adjuvant TMZ. Furthermore, patients with methylated MGMT show pseudoprogression more frequently. ${ }^{12}$ Until recently, it was not well established whether this latter finding was due to frequent tumor progression immediately after treatment in unmethylated MGMT and, thus, unresponsive tumors or due to a high incidence pseudoprogression as a consequence of higher sensitivity to treatment; we now know it is the latter. ${ }^{9}$ MGMT promoter status may predict pseudoprogression in $>90 \%$ of patients with methylated glioblastoma. An approximately $60 \%$ probability of early true tumor progression was observed in unmethylated MGMT promoter tumors. ${ }^{11}$

The higher rates of methylated MGMT promoter found in patients with pseudoprogression may be correlated with the efficacy of concurrent radiochemotherapy on residual tumor burden. MGMT methylation status may prove to be an important clinical factor in the evaluation of imaging-based changes early in the post-RT phase of treatment. Thus, we can speculate that methylated MGMT may be a good indicator of therapeutic response and a better prognosis, given that an increased overall survival has been observed in these patients.

\section{Incidence}

Although the occurrence of pseudoprogression has long been known, its real incidence has not yet been reported in a large series of patients, perhaps because only in the past few years has there been standardization of initial therapy. Some reports with a small number of cases described a variable incidence of pseudoprogression (Table). ${ }^{10,11,13-19}$ The wide variation in the incidence of pseudoprogression may be due to a lack of defined criteria for correct diagnosis of this entity, patient selection bias, and the variable response criteria that have been used. The association between pseudoprogression and
MGMT promoter status seems to be high. A 91.3\% probability of pseudoprogression in patients with methylated MGMT promoter tumors and a 59\% probability of early-progression disease in unmethylated MGMT promoter tumors have been described previously. Pseudoprogression has been reported to occur predominantly (in almost $60 \%$ of cases) within the first 3 months after completing treatment, but it may occur from the first few weeks to 6 months after treatment. ${ }^{11}$

\section{Pathophysiology}

Increased contrast enhancement and peritumoral edema following RT, with or without concomitant TMZ, may reflect tumor growth if the changes become stable. Alternatively, increased contrast enhancement and peritumoral edema that diminish with time are characteristic of pseudoprogression. Although it can occur following RT alone, pseudoprogression is widely believed to be more frequent following concomitant RT-TMZ. ${ }^{9}$ However, pseudoprogression can be associated with other chemotherapy regimens and has even been observed in cases in which chemotherapy-infused wafers were placed in the surgical cavity. By definition, pseudoprogression subsides without further treatment but, in some cases, appears to progress with time into radiation necrosis or treatmentrelated necrosis. The concept of therapy-induced necrosis and its radiologic manifestations of pseudoprogression should replace the outdated term "early necrosis." ${ }^{20}$ These types of necrotic lesions should not be considered strictly radionecrosis because they are included in the treatment effects, implying a potential difference in patient outcome. Effective treatment can involve disruption of the BBB, which facilitates passage of the drug and, thus, results in an enhancement of its activity. This damage to the BBB can persist for several months after treatment, showing an enhanced lesion that appears larger than before initiation of RT and thus simulates disease progression. ${ }^{20}$

Pathologically, pseudoprogression is found to correspond to gliosis and reactive radiation-induced changes without evidence of viable tumor. ${ }^{21}$ Pseudoprogression may represent an 


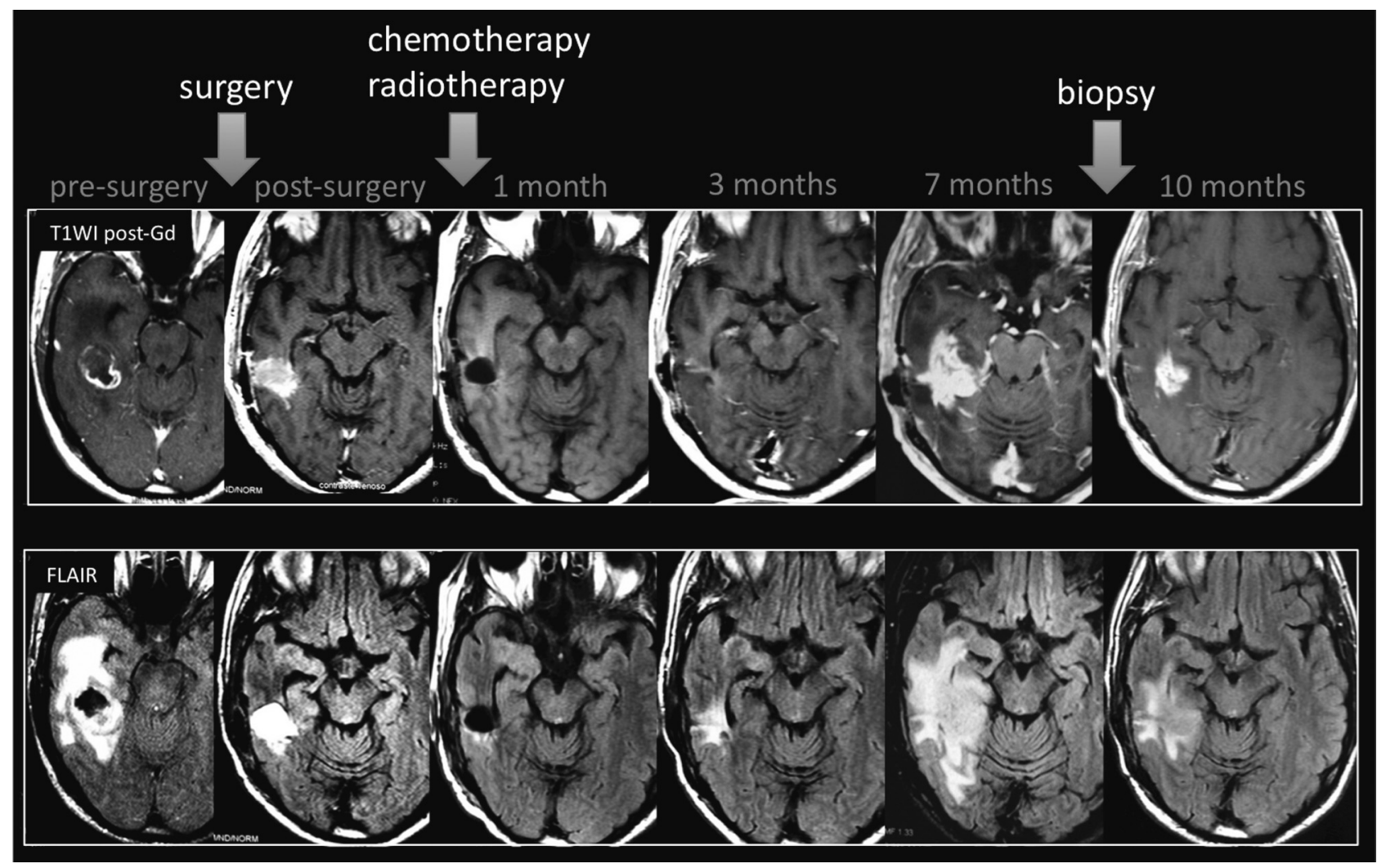

Fig 2. Pseudoprogression. A 63-year-old man with GBM. A follow-up MR imaging examination performed 7 months after RT-TMZ demonstrates increased lesion size. The histopathology samples (not shown) demonstrated a mixed tissue with treatment-related changes, associated with a few areas of viable tumor cells.

exaggerated response to effective therapy, involving early changes to the vascular endothelium and the $\mathrm{BBB},{ }^{9}$ as well as oligodendroglial injury leading to inflammation and increased permeability. The abnormal enhancement seen in these patients may be secondary to treatment-related cellular hypoxia, which results in the expression of hypoxia-regulated molecules from the tumor and surrounding cells, with subsequent increased vascular permeability. ${ }^{22}$

Pathologists should be aware of concerns regarding the correct diagnosis of this entity because the evaluation of these specimens can be challenging. The use of biopsy samples can be difficult due to the potential heterogeneity of these lesions and the findings of mixed tissue. Moreover, pathology of post-RT specimens may not be helpful in predicting outcome or in deciding on further management. ${ }^{19}$ Finally, there can be interpathologist variability in the interpretation of what constitutes a viable tumor versus treatment-related changes or gliosis in treated tumor samples (Fig 2). ${ }^{23}$

\section{Diagnosis}

Pseudoprogression may influence the clinical recommendation to continue with adjuvant chemotherapy rather than the change to a second-line therapy for recurrence. ${ }^{16}$ The diagnosis of pseudoprogression should depend on follow-up scans until an improved method is established. The terminology of pseudoprogression in some ways overlaps the classic definition of radiation necrosis, which also consists of marked enhancement in the tumor bed but without actual tumor; it is not incorrect to say that pseudoprogression represents a mild and self-limiting variant of treatment-related necrosis. ${ }^{5}$ This overlap in terminology is somewhat unavoidable, so care should be taken to focus more on the findings and, given the imprecision of the methods in common use, to avoid specific pronouncements regarding tumor-versus-radiation effect. At some time points, the distinction might be easier-for example, if an increased enhancement at the first post-RT MR imaging is observed, this is some sort of radiation effect and most likely will subside. However, early rapid progression cannot be ruled out and is merely the less probable diagnosis, not something that imaging findings can confidently establish.

Radiation necrosis typically occurs $18-24$ months posttreatment and has repeatedly been shown to be difficult to distinguish from recurrence. However, pseudoprogression is observed only in the first few months after treatment, much earlier than radiation necrosis. Some authors have suggested that the concept of therapy-induced necrosis and its radiologic manifestations of pseudoprogression should replace the outdated term "early radionecrosis"; we concur. ${ }^{20}$ Conventional MR imaging studies at follow-up examination may reveal increased contrast enhancement and peritumoral edema following treatment. Follow-up MR imaging in the assessment of the evolution of these lesions is useful for depicting pseudoprogression because the contrast enhancement portion of the lesion remains stable or diminishes with time. ${ }^{16}$ In some cases, true tumor progression is observed.

As mentioned previously, to date, no single imaging technique has been validated to recognize and adequately establish a diagnosis of pseudoprogression. ${ }^{14}$ Although the Macdonald Criteria have been widely used, false interpretations of tumor size increases on post-gadolinium-enhanced T1-weighted 


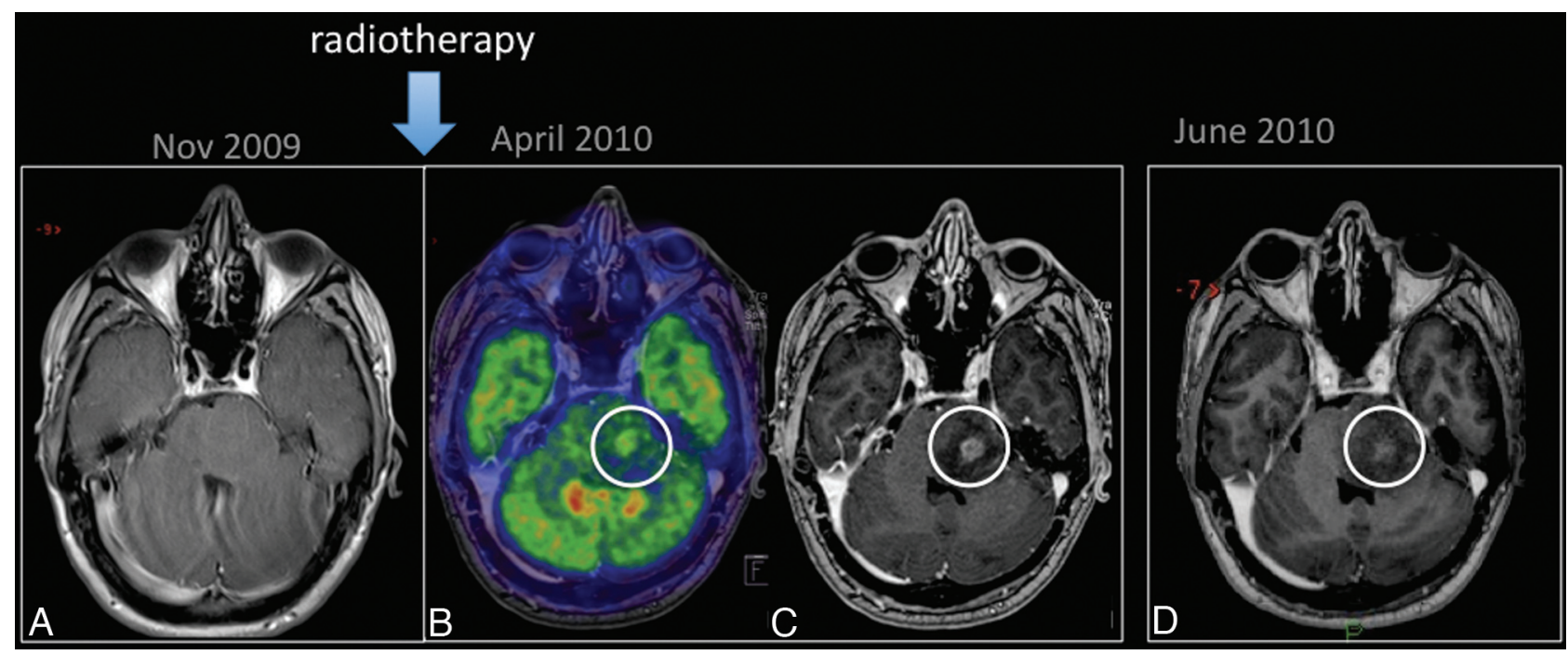

Fig 3. Pseudoprogression. A 25-year-old man with a low-grade glioma in the left aspect of the pons ( $A$, arrow) was treated with only RT. PET-MR imaging (B) showed hypermetabolism in the enhancing portion of the lesion (C). An MR imaging examination performed 1 month later $(D)$ shows a reduction in the enhancing portion of lesion.

imaging may occur. New MR imaging and/or PET tools are needed to characterize tumors before initiation of therapy, depict the changes that result from treatment, and be validated as biomarkers of treatment effectiveness. ${ }^{15}$ We will briefly review some of these potential new methods.

\section{Advanced MR Imaging Techniques}

DWI has been assessed to differentiate tumor progression and/or residual tumor from necrosis. ADC values were noted to be higher in necrotic tissue than in recurrent tumor tissue. However, available evidence for this imaging technique is limited due to the heterogeneity of tumor content, and differences in groups of patients do not imply high diagnostic performance. ${ }^{11}$ Other investigators using DTI have demonstrated a higher fractional anisotropy and decreased ADC values in the normal-appearing white matter adjacent to the edema in patients with radiation injury compared with patients with recurrent tumors. ${ }^{24}$ In short, neither DWI nor DTI provides sufficient information for differential diagnosis between pseudoprogression and true tumor progression. Both imaging modalities generally present heterogeneous signal intensities on DWI and ADC maps, with areas of reduced diffusion that can represent highly cellular tumor areas and/or inflammatory processes.

MR spectroscopy can depict structural degradation in cerebral tissue after RT before the development of symptoms or evidence of changes can be observed by conventional MR imaging. MR spectroscopy can reveal significant alteration in brain metabolites such as a slight decrease of NAA and variable changes in Cho. An extensive metabolic peak of lipids/lactate has been demonstrated in radiation-injured brain tissue as well. ${ }^{25}$ Notwithstanding, in most settings, the differential diagnosis between pseudoprogression and true disease progression based on MR spectroscopy findings is highly challenging. Both types of lesions can present with neuronal loss/dysfunction (low NAA), abnormal cellular membrane attenuation/ integrity (high Cho), and anaerobic metabolism (high lactate/lipids).

DSC is a surrogate marker for angiogenesis and has been used to assess brain tumor treatment response with high sensitivity for distinguishing residual/recurrent neoplasm from radiation brain injury. Percentage of signal intensity recovery, obtained from DSC data, is an imaging indicator of microvascular leakiness and is a significant variable to differentiate retrospectively whether a progressive enhancing lesion was due to recurrent tumor or radiation necrosis. ${ }^{25}$ rCBV values are also useful for differentiating treatment-related effects from viable tumor because they can provide evidence of neoangiogenesis in lesions. Recently, rCBV values have been used to predict pseudoprogression. ${ }^{15,22}$ Mangla et al ${ }^{15}$ evaluated rCBV values in patients with GBM before and 1 month after RTTMZ. In patients with pseudoprogression, there was a $41 \%$ mean decrease in rCBV, while cases of true tumor progression showed a $12 \%$ increase in $\mathrm{rCBV}$ from pretreatment to posttreatment. ROC revealed an area under the ROC curve of 0.85 , with $76.9 \%$ sensitivity and $85.7 \%$ specificity. Tsien et al ${ }^{21}$ also demonstrated a reduction in $\mathrm{rCBV}$ in patients with pseudoprogression by using a voxel-based analysis. In another study, authors warned that DSC could underestimate rCBV in progressive disease, due to rapid contrast extravasations into extravascular extracellular space. ${ }^{22}$ To reduce this limitation, a predose can be administered before the DSC sequence to reduce leakage of the contrast into the extracellular space. The authors also suggested using new contrast agents that do not extravasate owing to their larger molecular sizes. ${ }^{22}$

Permeability DSC is also a potential new tool for differential diagnosis between pseudoprogression and true tumor progression. Although no prospective study has examined this hypothesis, preliminary results with this new technique seem very promising, and a number of clinical trials are underway to better delineate the performance of all of the above techniques.

\section{PET}

FDG-PET can demonstrate differences in the analysis of areas of radiation injury and residual/recurrent brain tumors. However, the reported sensitivity and specificity are low or disputed in some studies. ${ }^{26,27}$ These limitations can be explained 
by the high glucose use in the brain, which results in high background activity, ${ }^{9}$ and by the fact that inflammatory processes can demonstrate high glucose metabolism on PET examinations. To our knowledge, no published data regarding the use of FDG-PET in the assessment of pseudoprogression are available (Fig 3).

In general, both tumor progression and pseudoprogression should be considered possibilities for focal enhancement that appears in the irradiated area within 6 months after completion of treatment. The differentiation of a recurrent/progressive tumor from radiation injury is often a radiologic dilemma, regardless of the imaging technique used. There is a growing necessity to perform standard management in these patients; however, no specific imaging characteristic findings are yet able to make such a differentiation. Potential methods for the future may include metabolic imaging either with MR imaging or PET, though even this might not distinguish active inflammation (which is highly metabolically active) from tumor. Alternatively, other features of the cancer such as hypoxia or proliferation might be feasible. Until such methods are available, however, most typically the clinical course, including imaging during a lengthy follow-up interval, enables the distinction of these 2 entities rather than specific imaging data. On some occasions, a brain biopsy may be needed; clearly, a noninvasive tool to differentiate these entities when a new enhancing lesion is first identified would be highly valuable. ${ }^{11}$

\section{Clinical Management}

Transient increases in contrast enhancement just after completion of chemoradiotherapy play an important role in the clinical management of patients with cancer because they complicate the ability of the physician to determine whether to continue with standard adjuvant chemotherapy or to switch to a second-line therapy for recurrence. Thus, the detection of pseudoprogression versus true progression is a critically important issue in oncology practice.

If a postchemoradiotherapy follow-up MR imaging examination demonstrates complete or partial response or stable disease (ie, smaller or stable tumor enhancement), maintenance of chemotherapy is typically continued. When enlargement occurs, then the treating physician does face a dilemma. If pseudoprogression is suspected, perhaps based on MGMT status and/or very early changes in imaging features in the first months posttreatment, ongoing chemotherapy with TMZ might be continued, with close monitoring. In clinically symptomatic patients, more options must be considered, including cessation of therapy, addition of anti-VEGF treatment, or even surgery. Identical symptoms can be observed in patients with true tumor progression and patients with pseudoprogression. ${ }^{5}$

\section{Pseudoresponse}

Antiangiogenic agents such as bevacizumab, an anti-VEGF antibody, and cediranib, a VEGF receptor tyrosine kinase inhibitor, have been tested in recent high-grade glioma treatment trials. These agents produce a rapid decrease in contrast enhancement with a high response rate and 6-month progression-free survival, but with rather modest effects on overall survival. ${ }^{28}$ Contrast-enhanced MR imaging was used as the basis for determining response in those trials; improvements

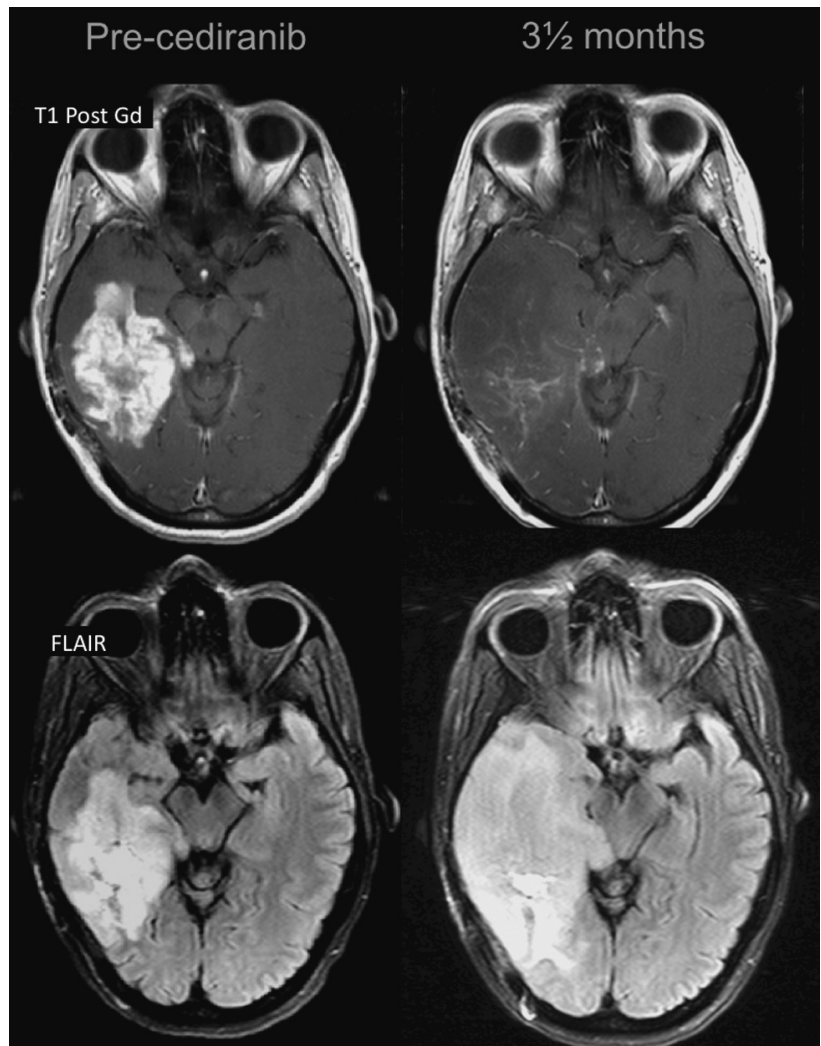

Fig 4. Pseudoresponse is characterized by a marked decreased in the enhancing portion of the lesion some months after initiation of treatment. However, in some such cases, the FLAIR sequence shows a clear expansion of the lesion.

were seen as early as 18 days after initiating therapy with bevacizumab and, in some patients, were associated with clinical improvements. ${ }^{29}$ In a small percentage of patients, a new pattern of progression has been identified ${ }^{30}$ : Enlargement of the nonenhancing portion of the lesion on T2-weighted and FLAIR sequences was observed in follow-up MR imaging examinations (Fig 4). Inhibition of angiogenesis may precede and even stimulate other routes of tumor dissemination that circumvent the effects of angiogenesis inhibition, the so-called vessel co-option. ${ }^{31}$ This appears particularly evident on diffusion MR imaging. ${ }^{32,33}$ This phenomenon may explain why the survival increases are modest at best, despite a tremendous response rate and progression-free survival. ${ }^{5}$

The early decrease in contrast enhancement suggests a change in vascular permeability, with a "normalization" of the $\mathrm{BBB}$, rather than a true tumor reduction, as being the underlying cause of the improvement. The rapid change can occur within hours of beginning therapy. Apparent responses to antiangiogenic therapy may be partly a result of normalization of abnormally permeable tumor vessels. ${ }^{34}$ Thus, the radiologic response should be interpreted with caution because a very brief response-perhaps lasting just a few days or weeks and therefore termed "pseudoresponse" - may be responsible for the imaging and clinical response (Fig 5). Very rapid results were also demonstrated with cediranib, which produced a normalization of the BBB as shown on MR imaging within 24 hours ${ }^{35}$ Reversibility of this vascular normalization, with rebound enhancement and edema, was noted when patients required a "drug holiday," mostly due to toxicity, with a "re- 


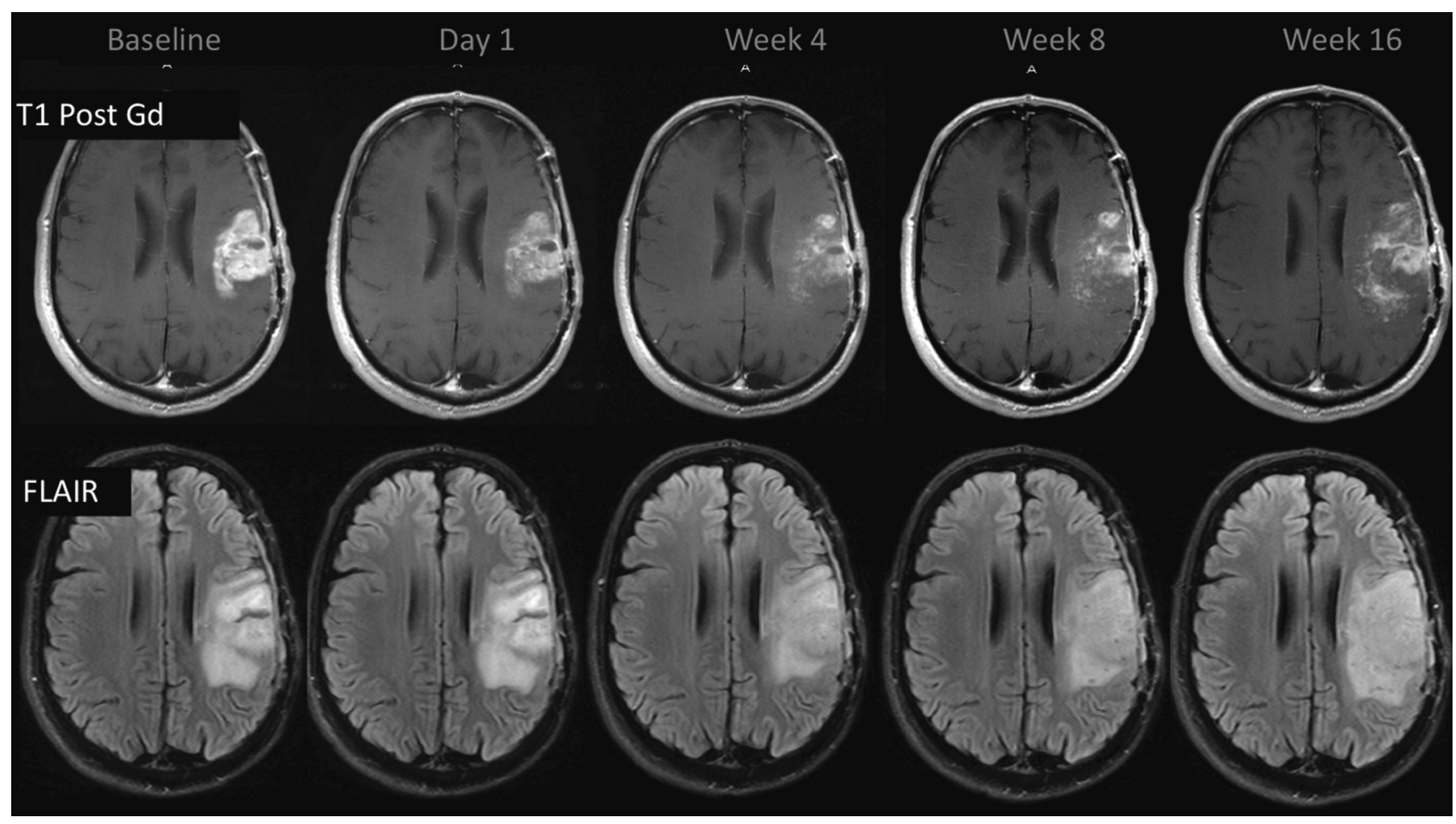

Fig 5. Pseudoresponse. A 47-year-old man with GBM. A reduction of the enhancing portion of the lesion is observed 1 day after initiation of cediranib treatment. Four weeks later, besides a continuing reduction in the enhancing portion, an expansion is observed in the FLAIR images. Expansions in both the enhancing area and abnormal hyperintense areas consistent with tumor progression were observed subsequently.

response" after restart. ${ }^{23}$ On the other hand, the degree of decreased enhancement to these anti-VEGF therapies after just 1 day of treatment correlated well with survival. ${ }^{35}$ This suggests that pseudoresponse may well be associated with a clinical benefit, just as pseudoprogression is also associated with clinical benefit.

In addition, whether a true antiglial effect or just a pseudoresponse is observed, normalization of the BBB and subsequent reduction in the vasogenic edema can result in an improvement of symptoms, a reduction of steroid dependence, and an improvement of brain function and quality of life, bringing clinical benefits to patients. ${ }^{5,23}$

\section{Acknowledgments}

The authors thank Tracy Batchelor and Elizabeth Gerstner for their help in editing the manuscript.

\section{Conclusions}

Pseudoprogression and pseudoresponse are abnormalities that have been described following high-grade tumor treatment, and remarkably both appear to be associated with future favorable patient outcome. Both phenomena appear to be best diagnosed through follow-up scans because no established method of imaging is yet capable of yielding a definitive diagnosis of true tumor versus enhancement changes due to other reasons. DSC and other methods appear promising but require further testing in the multicenter setting.

\section{References}

1. Stupp R, Mason WP, van den Bent MJ, et al. Radiotheraphy plus concomitant and adjuvant temozolomide for glioblastoma. N Engl J Med 2005;352:987-96

2. Macdonald DR, Cascino T, Schold SJ, et al. Response criteria for phase II studies of supratentorial malignant glioma. J Clin Oncol 1990;8:1277-80
3. Wen PY, Macdonald DR, Reardon DA, et al. Updated response assessment criteria for high-grade gliomas: response assessment in neuro-oncology working group. J Clin Oncol 2010;28:1963-72

4. Sorensen AG, Batchelor TT, Wen PY, et al. Response criteria for glioma. Nat Clin Pract Oncol 2008;5:634-44

5. Brandsma D, van den Bent MJ. Pseudoprogression and pseudoresponse in the treatment of gliomas. Curr Opin Neurol 2009;22:633-38

6. Kumar AJ, Leeds NE, Fuller GN, et al. Malignant gliomas: MR imaging spectrum of radiation therapy- and chemotherapy-induced necrosis of brain after treatment. Radiology 2000;217:377-84

7. Ulmer S, Braga TA, Barker FG 2nd, et al. Clinical and radiographic features of peritumoral infarction resection of glioblastoma. Neurology 2006:67:1668-70

8. de Wit MC, de Bruin HG, Eijkenboom W, et al. Immediate post-radiotherapy changes in malignant glioma can mimic tumor progression. Neurology 2004;63:535-37

9. Brandsma D, Stalpers L, Taal W, et al. Clinical features, mechanisms, and management of pseudoprogression in malignant glioma. Lancet Oncol 2008;9:453-61

10. Taal W, Brandsma D, de Bruin HG, et al. Incidence of early pseudo-progression in cohort of malignant glioma patients treated with chemoirradiation with temozolomide. Cancer 2008;113:405-10

11. Brandes AA, Franceschi E, Tosoni A, et al. MGMT promoter methylation status can predict the incidence and outcome of pseudoprogression after concomitant radiochemotherapy in newly diagnosed glioblastoma patients. J Clin Oncol 2008;26:2192-97

12. Brandes AA, Tosoni A, Franceschi E, et al. Recurrence pattern after temozolomide concomitant with and adjuvant to radiotherapy in newly diagnosed patients with glioblastoma: correlation with MGMT promoter methylation status. J Clin Oncol 2009;27:1275-79

13. Chamberlain MC. Pseudoprogression in glioblastoma. J Clin Oncol 2008;26:4359

14. Chaski C, Neyns B, Michotte A, et al. Pseudoprogression after radiotherapy with concurrent temozolomide for high-grade glioma: clinical observations and working recommendations. Surg Neurol 2009;72:423-28

15. Mangla R, Singh G, Ziegelitz D, et al. Changes in relative cerebral blood volume 1 month after radiation-temozolomide therapy can help predict overall survival in patients with glioblastoma. Radiology 2010;256:575-84

16. Sanghera P, Perry J, Sahgal A, et al. Pseudoprogression following chemoradiotherapy for glioblastoma multiforme. Can J Neurol Sci 2010;37:36-42

17. Clarke JL, Abrey LE, Karimi S, et al. Pseudoprogression (PsPr) after concurrent radiotherapy (RT) and temozolomide (TMZ) for newly diagnosed glioblastoma multiforme (GBM) [abstract]. J Clin Oncol 2008;26:2025

18. Gerstner ER, McNamara MB, Norden AD, et al. Effect of adding temozolomide 
to radiation therapy on the incidence of pseudo-progression. J Neurooncol 2009;94:97-101

19. Jefferies $\mathrm{S}$, Burton $\mathrm{K}$, Jones $\mathrm{P}$, et al. Interpretation of early imaging after concurrent radiotherapy and temozolomide for glioblastoma [abstract]. Clin $\mathrm{On}$ col 2007; 19:S33

20. Brandes AA, Tosoni A, Spagnolli F, et al. Disease progression or pseudoprogression after concomitant radiochemotherapy treatment: pitfalls in neurooncology. Neuro Oncol 2008;10:361-67. Epub 2008 Apr 9

21. Tsien C, Galbán CJ, Chenevert TL, et al. Parametric response map as an imaging biomarker to distinguish progression from pseudoprogression in highgrade glioma. J Clin Oncol 2010;28:2293-99

22. Gahramanov S, Raslan AM, Muldoon LL, et al. Potential for differentiation of pseudoprogression from true tumor progression with dynamic susceptibility-weighted contrasted-enhanced magnetic resonance imaging using feruxomytol vs. gadoteridol: a pilot study. Int J Radiat Oncol Biol Phys 2011;79:514-23

23. Clarke JL, Chang S. Pseudoprogression and pseudoresponse: challenges in brain tumor imaging. Curr Neurol Neurosci Rep 2009;9:241-46

24. Sundgren PC, Fan X, Weybright $P$, et al. Differentiation of recurrent brain tumor versus radiation injury using diffusion tensor imaging in patients with new contrast enhancing lesions. Magn Reson Imaging 2006;24:1131-42

25. Sundgren PC. MR spectroscopy in radiation injury. AJNR Am J Neuroradiol 2009;30:1469-76

26. Ricci PE, Karis JP, Heiserman JE, et al, Differentiating recurrent tumor from radiation necrosis: time for re-evaluation of positron emission tomography? AJNR Am J Neuroradiol 1998;19:407-13
27. Hustinx R, Pourdehnad M, Kaschten B, et al. PET imaging for differentiating recurrent brain tumor from radiation necrosis. Radiol Clin North Am 2005; $43: 35-47$

28. Batchelor TT, Sorensen AG, di Tomaso E, et al. AZD2171, a pan-VEGF receptor tyrosine kinase inhibitor, normalizes tumor vasculature and alleviates edema in glioblastoma patients. Cancer Cell 2007;11:83-95

29. Pope WB, Lai A, Nghiemphu P, et al. MRI in patient with high-grade gliomas treated with bevacizumab and chemotherapy. Neurology 2006;66:1258-60

30. Norden AD, Young GS, Setayesh K, et al. Bevacizumab for recurrent malignant gliomas: efficacy, toxicity, and patterns of recurrence. Neurology 2008;70:779-87

31. Jain RK, Duda DG, Willett CG, et al. Biomarkers of response and resistance to antiangiogenic therapy. Nat Rev Clin Oncol 2009;6:327-38

32. Gerstner ER, Chen PJ, Wen PY, et al. Infiltrative patterns of glioblastoma spread detected via diffusion MRI after treatment with cediranib. Neuro Oncol 2010;12:466-72

33. Pope WB, Kim HJ, Huo J, et al. Recurrent glioblastoma multiforme: ADC histogram analysis predicts response to bevacizumab treatment. Radiology 2009;252:182-89

34. Gerstner ER, Duda DG, di Tomaso E, et al. VEGF inhibitors in the treatment of cerebral edema in patients with brain cancer. Nat Rev Clin Oncol 2009;6:229-36

35. Sorensen AG, Batchelor TT, Zhang WT, et al. A "vascular normalization index" as potential mechanistic biomarker to predict survival after a single dose of cediranib in recurrent glioblastoma patients. Cancer Res 2009;69:5296-300 